\title{
EVOLUCIÓN A UN AÑO DE LOS EFECTOS ADVERSOS, EN UNA COHORTE DE PACIENTES CON IMPLANTE SUBDÉRMICO DE DESOGESTREL
}

\author{
Adolfo Patiño V. ${ }^{1}, M$. del Rosario Ruelas $C^{2}{ }^{2}$, Enrique Villarreal R. ${ }^{3}$, Lidia Martínez G. ${ }^{3}$, \\ Liliana Galicia R. ${ }^{3}$, Emma Rosa Vargas D. ${ }^{3}$ \\ ${ }^{1}$ Unidad de Medicina Familiar № 16. 르oordinación de Salud Reproductiva, ${ }^{3}$ Unidad de Investigación Epidemiológica y \\ en Servicios de Salud, Instituto Mexicano del Seguro Social, Querétaro, México.
}

\section{RESUMEN}

Objetivo: El propósito de la investigación fue evaluar la evolución a un año los efectos adversos en una cohorte de pacientes con implante subdérmico de desogestrel. Método: Se incluyeron pacientes portadoras de implante que tenían como mínimo tres meses de uso, previo consentimiento informado. Se realizaron 4 mediciones en forma trimestral, registrándose los efectos más frecuentes referidos por la paciente. El análisis incluyó porcentajes, promedios e intervalos de confianza al 95\%. Resultados: Se estudiaron 50 pacientes, los efectos adversos presentados con mayor frecuencia en el primer trimestre fueron alteraciones del ciclo $48 \%$, mastalgia $46 \%$, amenorrea $40 \%$, mareo $40 \%$, cefalea $40 \%$, náuseas $30 \%$, acné $28 \%$, aumento de peso $26 \%$ y dolor local $10 \%$. Se observó al final del tercer trimestre reducción de la cefalea, mastalgia y mareo $(p<0,05)$; para el cuarto trimestre las náuseas y las alteraciones del ciclo $(p<0,05)$. El $11,1 \%$ de las pacientes desertaron del método al final del estudio. Se observó que el uso del desogestrel tuvo un valor estadísticamente significativo para el control de la dismenorrea $(p<0,05)$ al final del cuarto trimestre de uso. Conclusión: Los implantes de desogestrel tienen efectos adversos importantes en los primeros meses de uso para disminuir la mayoría de ellos al final del primer año.

\section{PALABRAS CLAVES: Desogestrel, implante subdérmico, efectos adversos}

\section{SUMMARY}

Objective: To determine the adverse effects of implants subskin of desogestrel. Method: A descriptive longitudinal study was made in a cohort carrying patients of implants that they had as minimum three months of use, previous informed consent were included in the study. Four measurements were made in quarterly form, registering the most frequent effects, referred by the patient. It was used descriptive statistic and confidence intervals. Results: It was study 50 patients. The presented effects with greater percentage in the first trimester were: alterations of cycle $48 \%$, mastalgia $46 \%$, amenorrhoea $40 \%$, dizzies $40 \%$, headache $40 \%$, nauseas $30 \%$, acne $28 \%$, increase of weight $26 \%$ and local pain $10 \%$. It was observed at the end of the third quarterly reduction of the statistically significant effects of the headache, mastalgia, dizzies $(p<0.05)$, for the fourth trimester nauseas and alterations of the cycle $(p<0.05)$. The $11.1 \%$ of the patients deserted of the method at the end of the study. It was observed that the use of desogestrel had a significant statistic for the control of the dismenorrea with $p<0.05$ at the end of the fourth trimester of use. Conclusions: You implant them of desogestrel have adverse effects important in the first's months of use to diminish most of them at the end of the first year.

KEY WORDS: Desogestrel, implants subskin, adverse effects 


\section{INTRODUCCIÓN}

Los implantes han surgido como una necesidad en la búsqueda del anticonceptivo ideal, el cual debe tener alta seguridad, mínimos efectos colaterales, reversible, cómodo, fácil de usar y ser de larga duración $(1,2)$. Están constituidos por una goma de silicona, sobre la cual va depositada la progestina, concretamente levonorgestrel 0 desogestrel $(3,4)$. El desogestrel como método de planificación familiar surgió en 1996, contiene un promedio de 70.000 ciclos de 28 días y una duración de 3 años $(5,6,7)$.

Entre las principales ventajas de los implantes se ha identificado la liberación estable del fármaco y el evitar fallos por parte de la usuaria, lo que se asocia con seguridad y confianza $(3,8)$. Pero también se ha observado que ocasionan efectos adversos no deseados en los primeros meses de uso, entre los más frecuentes, se encuentran alteraciones del ciclo menstrual, orden de frecuencia, sangrado frecuente e irregular, goteos u oligomenorrea, sangrado prolongado, amenorrea, mareo, náuseas, cefalea y dolor local $(3,9,10)$. Otros efectos de las progestinas solas por su efecto androgénico son el aumento de peso, acné e hirsutismo (11).

Ante este panorama y considerando que el implante subdérmico de desogestrel es utilizado en la actualidad como método de planificación familiar, se planteó como objetivo evaluar la evolución de los efectos secundarios a un año en una cohorte de pacientes.

\section{MATERIAL Y MÉTODO}

Se realizó un estudio de tipo longitudinal descriptivo en una cohorte de mujeres en edad fértil, portadoras de implante subdérmico como método de planificación familiar, atendidas en unidades de medicina familiar, de la Ciudad de Querétaro, del Instituto Mexicano del Seguro Social, durante el periodo de enero del 2004 a enero del 2005. Se incluyeron aquellas pacientes con uso mínimo de tres meses del implante subdérmico. El tamaño de la muestra correspondió a 50 pacientes con pérdida final de 5 por retiro del método, la cohorte se integró a partir de las pacientes que acudían regularmente a los módulos de planificación familiar, realizándoles seguimiento domiciliario cuando no acudieran a consulta para la recolección total de la información de los trimestres. Los datos se recabaron trimestralmente mediante un cuestionario que incluía los efectos adversos del método, referidos por la paciente en forma trimestral. El análi- sis estadístico incluyó porcentajes, medias, desviación estándar, diferencia de porcentajes para dos poblaciones e intervalos de confianza al $95 \%$.

Este trabajo contó con la aprobación del Comité Local de Investigación y con la autorización escrita de las pacientes participantes.

\section{RESULTADOS}

Se estudiaron 50 pacientes portadoras de implante subdérmico de desogestrel, con deserción de 5 pacientes al final del año de seguimiento. La edad promedio fue de $26,3 \pm 4,5$ años, $54 \%$ con escolaridad de nivel medio superior, $92 \%$ casadas y $60 \%$ dedicadas al hogar.

El $40 \%$ fueron primigestas y $32 \%$ nuligestas, la mayoría de las pacientes cursaban con ciclos menstruales normales, $8 \%$ presentaba irregularidades y $14 \%$ refirió dismenorrea preimplante la cual disminuyó al final del primer año de uso $(p<0,05)$.

En el primer trimestre, las alteraciones del ciclo menstrual, manifestado por sangrado continuo, goteo o manchado fueron las más frecuentes, $48 \%$ (IC 95\%, 36-60); en el segundo trimestre de uso, los efectos más frecuentes continuaron siendo las alteraciones del ciclo, 42\% (IC 95\%, 31-54); en el tercer trimestre la amenorrea fue la alteración que se reportó con mayor porcentaje, 36\% (IC 95\%, 25-48); y en el cuarto trimestre el total de los efectos adversos disminuyeron, persistiendo la amenorrea, 35\% (IC 95\%, 24-47) (Tabla I).

La disminución de los efectos adversos se observó a partir del tercer trimestre, siendo estadísticamente significativos para alteraciones del ciclo menstrual, cefalea, mastalgia, y mareo; y para el cuarto trimestre el acné y náuseas (Tabla II).

El número de síntomas presentados por las pacientes según trimestre esta representado en la Tabla III, observándose disminución progresiva de acuerdo al tiempo de uso.

\section{DISCUSIÓN}

Las alteraciones del ciclo menstrual son los principales efectos adversos que ocasiona el desogestrel en implantes subdérmicos de liberación prolongada, reportándose hasta un $50 \%$ en los primeros tres meses de su uso para declinar al $30 \%$, después de los seis meses la prevalencia va disminuyendo hasta los primeros dos años $(2,8,12,13)$. En el presente estudio estas cifras fueron similares en los primeros tres meses observando una disminución porcentual al final del primer año. 
Tabla I

EFECTOS ADVERSOS POR TRIMESTRE EN PACIENTES CON IMPLANTE SUBDÉRMICO

\begin{tabular}{|c|c|c|c|c|c|c|c|c|}
\hline \multirow[t]{2}{*}{ Efectos adversos } & \multicolumn{2}{|c|}{$\begin{array}{c}1^{e r} \text { trimestre } \\
n=50\end{array}$} & \multicolumn{2}{|c|}{$\begin{array}{c}2^{\circ} \text { trimestre } \\
n=49\end{array}$} & \multicolumn{2}{|c|}{$\begin{array}{c}3^{e r} \text { trimestre } \\
n=47\end{array}$} & \multicolumn{2}{|c|}{$\begin{array}{c}4^{\circ} \text { trimestre } \\
n=45\end{array}$} \\
\hline & $\%$ & IC & $\%$ & $I C$ & $\%$ & IC & $\%$ & IC \\
\hline Alteraciones del ciclo & 48,0 & $36-60$ & 42,7 & $31-54$ & 29,7 & $19-41$ & 26,6 & $16-37$ \\
\hline Mastalgia & 46,0 & $34-58$ & 30,6 & $20-41$ & 19,1 & $10-29$ & 8,5 & $2-15$ \\
\hline Amenorrea & 40,0 & $29-51$ & 38,7 & $27-50$ & 36,1 & $25-48$ & 35,1 & $24-47$ \\
\hline Mareo & 40,0 & $29-51$ & 28,5 & $18-39$ & 21,2 & $11-31$ & 11,1 & 3-19 \\
\hline Cefalea & 40,0 & $29-51$ & 28,5 & $18-39$ & 12,7 & $5-21$ & 4,4 & $1-9$ \\
\hline Náuseas & 30,0 & $19-41$ & 20,4 & $11-30$ & 17,0 & $8-26$ & 8,8 & $2-16$ \\
\hline Acné & 28,0 & $18-38$ & 24,4 & $14-34$ & 17,0 & $8-26$ & 13,3 & $5-22$ \\
\hline Aumento de peso & 26,0 & $16-36$ & 26,5 & $16-37$ & 25,5 & $15-36$ & 20,0 & $10-30$ \\
\hline Dolor & 10,0 & $3-17$ & 8,1 & $2-14$ & 6,3 & $0-12$ & 6,6 & $1-13$ \\
\hline
\end{tabular}

$\mathrm{IC}=$ Intervalo de confianza al $95 \%$.

La amenorrea es otra de las alteraciones que se observa con mayor frecuencia desde primer año, persistiendo hasta los dos años de uso (2,8,11-14). Se observó en el estudio que en los primeros tres meses se presenta el mismo comportamiento y persiste al final del primer año.

Con respecto a dolor de mama, se ha reportado en un $29,9 \%$ en los primeros seis meses $(4,11)$, sin embargo se encontraron cifras superiores a lo reportado en el primer trimestre declinando a un $8,5 \%$ en el $4^{\circ}$ trimestre.

El mareo se presentó en un tercio de la población en los primeros seis meses que conforme avanza el tiempo este declina $(4,10,11)$. En el estudio se observó un mayor porcentaje en los primeros tres meses y con una persistencia mínima hasta el final del primer año.

La cefalea es uno de los efectos que más persisten durante el primer año $(4,10,11)$ en el estudio se encontraron resultados similares casi en la mitad de la población, disminuyendo conforme al tiempo de uso.

Una mínima proporción de las pacientes que utilizan implantes subdérmicos de progestinas presentan nauseas en el primer año de su utilización $(10,11)$, en este estudio fueron superiores en los primeros tres meses, y posteriormente similares a la literatura encontrada (3-12\%).

El dolor local en el sitio de la inserción y las infecciones se reportan hasta un $5 \%(2,8,10,12,15)$. No se reportaron datos de infección y solo una mínima parte refirió dolor local.

Con respecto a la actividad androgénica el aumento de peso esta presente entre $6-30 \%$ de las usuarias $(4,10,11,13)$. El comportamiento de las usuarias fue similar a lo encontrado en la literatura.

Tabla II

DISMINUCIÓN DE LOS EFECTOS ADVERSOS CON IMPLANTE SUBDÉRMICO EN RELACIÓN AL PRIMER TRIMESTRE

\begin{tabular}{|c|c|c|c|c|c|c|}
\hline \multirow[t]{2}{*}{ Efectos adversos } & \multicolumn{2}{|c|}{$\begin{array}{c}2^{\circ} \text { trimestre } \\
n=49\end{array}$} & \multicolumn{2}{|c|}{$\begin{array}{c}3^{\text {er }} \text { trimestre } \\
n=47\end{array}$} & \multicolumn{2}{|c|}{$\begin{array}{c}4^{\circ} \text { trimestre } \\
n=45\end{array}$} \\
\hline & $Z$ & $p$ & $Z$ & $p$ & $Z$ & $p$ \\
\hline Alteraciones del ciclo & 0,26 & 0,60 & 3,37 & 0,06 & 4,58 & 0,03 \\
\hline Mastalgia & 2,48 & 0,11 & 7,90 & 0,00 & 16,03 & 0,00 \\
\hline Cefalea & 1,43 & 0,23 & 9,16 & 0,00 & 16,83 & 0,00 \\
\hline Mareo & 0,02 & 0,23 & 3,98 & 0,04 & 10,19 & 0,00 \\
\hline Náuseas & 1,21 & 0,27 & 2,26 & 0,13 & 7,20 & 0,00 \\
\hline Acné & 0,16 & 0,69 & 1,67 & 0,19 & 3,07 & 0,07 \\
\hline Aumento de peso & 0,00 & 0,95 & 0,00 & 0,95 & 0,48 & 0,48 \\
\hline Amenorrea & 0,11 & 0,90 & 1,39 & 0,23 & 0,20 & 0,65 \\
\hline Dolor local & 0,00 & 0,97 & 2,08 & 0,78 & 0,05 & 0,83 \\
\hline
\end{tabular}

Prueba de diferencia de porcentajes para dos poblaciones. 
Tabla III

PORCENTAJE DE PACIENTES CON NÚMERO DE EFECTOS ADVERSOS POR TRIMESTRE

\begin{tabular}{lcccc}
\hline $\begin{array}{l}\text { Número de efectos } \\
\text { adversos }\end{array}$ & $\begin{array}{c}\text { Primer trimestre } \\
\%\end{array}$ & $\begin{array}{c}\text { Segundo trimestre } \\
\%\end{array}$ & $\begin{array}{c}\text { Tercer trimestre } \\
\%\end{array}$ & \multicolumn{2}{c}{ Cuarto trimestre } \\
$\%$ & $n=47$ & $n=45$ \\
\hline Ninguno & $n=50$ & $n=49$ & 20,7 & 20,7 \\
Uno & 4,0 & 4,0 & 18,8 & 18,8 \\
Dos & 16,0 & 20,3 & 29,2 & 29,2 \\
Tres & 18,0 & 23,3 & 12,5 & 12,5 \\
Cuatro & 14,0 & 14,1 & 8,3 & 8,3 \\
Cinco & 6,0 & 12,1 & 4,2 & 4,2 \\
Seis & 14,0 & 12,1 & 6,3 & 6,3 \\
Siete & 14,0 & 12,1 & 0,0 & 0,0 \\
Ocho & 10,0 & 2,0 & 0,0 & 0,0 \\
\end{tabular}

El acné esta presente en más de una cuarta parte de la población referida $(4,10,11)$. Las cifras fueron similares en el presente estudio, observándose con mayor porcentaje en los primeros tres meses.

Se ha descrito que las pacientes que presentaban dismenorrea severa, con el uso de este método reducen sustancialmente su sintomatología (13). La reducción de esta se comportó de manera igual en casi la totalidad de las pacientes del estudio.

Se ha encontrado una mínima deserción hacia el uso del método $(5,3 \%)$ en donde a mayor tiempo de uso menor es la deserción. Las principales causas son las irregularidades en el ciclo (sangrados continuos) (8). Para el estudio las causas fueron las mismas pero observándose con mayor porcentaje $(11,1 \%)$.

Se encontró en este estudio un número de síntomas presentados por las pacientes; estos se presentaron con mayor proporción durante el primer trimestre disminuyendo con relación al tiempo de uso, esto es debido al efecto del desogestrel.

Se puede concluir que el uso de desogestrel en implantes de liberación prolongada tiene efectos adversos importantes en los primeros meses de uso, con reducción de los mismos conforme transcurre el tiempo. Se observó que en las pacientes con dismenorrea tiene un efecto benéfico para el control de la misma.

\section{BIBLIOGRAFÍA}

1. Vasconcelos AM, Unda UM. Implantes anticonceptivos de levonorgestrel. Realidades y controversias de un método anticonceptivo actual. Ginecol Obstet Mex 1996;64(11):498-502.

2. Baird DT, Glasier AF. Science medicine and the future: Contraception 1999;319(7215):969-72.
3. Morales DO, Vega SA, Fuentes SG, Pérez PG. Anticoncepción postaborto. Ginecol Obstet Mex 1998;66(2):65-8.

4. Sukjai B, Surasak T. Implanon use in Thai women above the age of 35 years. Contraception 2004; 489-91.

5. Croxatto HB, Makarainen L. The pharmacodynamics and efficacy of implanon. An overview of the data. Contraception 1998;58(6 Suppl):91s-97s.

6. Huber J. Pharmacokinetics of implanon. Contraception 1998;58:85S-90S.

7. Rene W, Agaath VB, Peter S, Johannes H. Pharmacokinetics of etonogestrel released from the contraceptive implant implanon. Contraception 1998;58: 283-88.

8. Lozano BA. La contracepción hormonal. Rev Med Integral 1999;33(4). Disponible en: http://avivasg.eresmas.com/newsacho.htm.

9. Glasier A. Implantable contraceptives for women: effectiveness, discontinuation rates, return of fertility, and outcome of pregnancies. Contraception 2002; 65:29-37.

10. Biran A, Tjeerd K, Affandi V, Korver T, Geurts TB, Paul GH, Coelingh B. A pilot efficacy study with a single-rod contraceptive implant (implanon) in 200 Indonesian women treated for $\leq 4$ years. Contraception 1999;59(3):167-74.

11. Urbancsek J. An integrated analysis of nomenstrual adverse events with implanon. Contraception 1998 58:109-15.

12. Herjan JT, Coelingh B. Presentation of clinical data on implanon. Contraception 1998;75S-77S.

13. Varma R, Mascarenhas L. Endometrial effects of etonogestrel (implanon) contraceptive implant. Obstet Gynecol 2001;13:335-41.

14. Brache V. Nonmenstrual adverse events during use of implantable contraceptives for women: data from clinical trials. Contraception 2002;65:63-74.

15. Mascarenhas L. Insertion and removal of implanon. Contraception 1998;58(6 Suppl):79S-83S. 